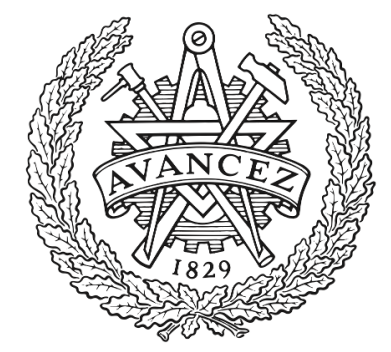

CHALMERS

UNIVERSITY OF TECHNOLOGY

\title{
Design of Spiral Constellations for Phase Noise Channels
}

Downloaded from: https://research.chalmers.se, 2023-04-26 14:39 UTC

Citation for the original published paper (version of record):

Ugolini, A., Piemontese, A., Eriksson, T. et al (2019). Design of Spiral Constellations for Phase

Noise Channels. IEEE Wireless Communications and Networking Conference, WCNC, 2019-April. http://dx.doi.org/10.1109/WCNC.2019.8885476

N.B. When citing this work, cite the original published paper. 


\title{
Design of Spiral Constellations for Phase Noise Channels
}

\author{
Alessandro Ugolini ${ }^{\dagger}$, Amina Piemontese ${ }^{\ddagger}$, Thomas Eriksson ${ }^{\ddagger}$, and Changhao Du* \\ $\dagger$ Department of Engineering and Architecture, University of Parma, Parma, Italy \\ $\ddagger$ Department of Electrical Engineering, Chalmers University of Technology, Gothenburg, Sweden \\ * Laboratory for Communication and Network, Beijing Institute of Technology, China.
}

\begin{abstract}
In this paper, we consider the design of spiral constellations for channels affected by phase noise. The strength of the proposed constellations resides both on the performance and on the extreme simplicity of the design. The symbols can in fact be expressed in analytical form, and are uniquely defined through a single parameter that accounts for the phase and thermal noise variances. The performance of the proposed constellations are assessed in terms of information rate and error rate. Despite their simplicity, the new spiral constellations have excellent performance, especially when the constellation size grows large.
\end{abstract}

\section{INTRODUCTION}

A possible answer to the increasing demand for high datarate transmission over band-limited channels is the use of high order constellations, whose sensitivity to channel imperfections is very critical. High order modulation schemes are susceptible to phase noise $(\mathrm{PN})$ that arises from local oscillator instabilities. To improve the performance, a properly designed constellation can be employed.

The problem was addressed for example by Foschini et al. in [1] already in the early 70s. In this work, an approximate maximum likelihood detector was derived for a memoryless PN channel, and constellations that optimize its symbol error probability were obtained. More recently, in [2], constellation points are optimized by maximizing the approximate mutual information using a simulated annealing algorithm. In [3], the problem of finding the optimal constellation for PN channels is investigated by considering optimization formulations based on error rate and mutual information. In [4], the performance of high order amplitude-phase shift keying (APSK) constellations in PN channels are compared to the conventional phase shift keying (PSK) and quadrature amplitude modulation (QAM) constellations. Spiral constellations, called spiral QAM, have been considered for PN channels in [5].

Although it has been well investigated in the past, the problem of finding a good constellation robust to PN is far from being solved. The main drawback of the design approaches in the literature is the complexity, since they require the optimization of several parameters and some of them produce unstructured constellations that lack flexibility.

In this paper, we propose new spiral constellations for PN channels, whose points are properly located along an Archimedean spiral. The choice of this particular spiral is motivated by its property of constant radial distance between spiral laps. The proposed constellations have excellent performance and are very simple to design. The constellation symbols can in fact be expressed in analytical form, and are uniquely defined through a single parameter, therefore they are very versatile. We show that the new spiral constellations allow to achieve very high information rate (IR), and they are suitable for application in practical coded systems adopting state-of-the-art channel codes.

The paper is organized as follows. Section II describes the adopted system model. Section III presents the proposed constellation design. Section IV details the considered performance metrics. Section V presents several numerical results and comparisons. Finally, Section VI concludes the paper.

\section{System MODEL}

Let us consider the following model for the received sample,

$$
y_{k}=x_{k} e^{j \phi_{k}}+w_{k},
$$

where $x_{k}$ is the transmitted symbol, $\phi_{k}$ is a PN sample, assumed to have a zero-mean Gaussian distribution with variance $\sigma_{\phi}^{2}$, and $w_{k}$ is a complex additive white Gaussian noise (AWGN) term, with variance $\sigma_{w}^{2}$. The symbol $x_{k}$ can assume any point in the $M$-point signal constellation $\left\{c_{m}, m \in\right.$ $\{1, \ldots, M\}\}$. The PN in (1) represents the residual phase error that results from tracking and compensating the phase of the received signal using an estimator. In general, the PN samples may be correlated, however, in order to simplify the analysis, we assume that an ideal estimator is used that removes any correlation [3]. This is a reasonable assumption when PN has been tracked by resorting, for example, to pilot symbols.

\section{SPIRAl CONSTEllations DESIGN}

In this section, we describe the proposed spiral constellations. An Archimedean spiral is defined by the following equation

$$
s(t)=t e^{j t} .
$$

We propose to place the constellation points along the spiral, according to the following rule,

$$
c_{m}=t_{m} e^{j t_{m}} \quad m=1, \ldots, M,
$$

where $M$ is the cardinality of the desired constellation. In the following, we describe how to choose the parameters $t_{m}$. We first consider the AWGN case, then we describe the construction in the case of $\mathrm{PN}$. 


\section{A. Spiral Constellations for the AWGN Channel}

The way to place constellation points on a spiral when the only impairment is the AWGN is to choose the points regularly along the spiral, so that the distance between consecutive symbols is the same. With this aim, we formulate the equation for the constellation points as explained by the following theorem, proved in Appendix A.

Theorem 1. If we place constellation points on the spiral according to the equation $t_{m}=\sqrt{2 D m}$, we will get a distance between consecutive points that is approximately equal to $D$, for all points. If we set $D=2 \pi$, the consecutive laps of the spiral are at the same distance $D$ as the points along the spiral.

The final expression for placing the points is then $t_{m}=$ $\sqrt{4 \pi m}$. This definition approximately leads to constant distances $2 \pi$ between consecutive points along the spiral and between consecutive laps of the spiral. The constellation points are then normalized such that the resulting constellation has unitary energy.

\section{B. Spiral Constellations in Phase Noise}

We extend the construction of a spiral constellation to take into account the presence of PN. The proposed construction is based on the observation that the PN affects more severely symbols with higher magnitude. To see this, let us start from (1). For small $\phi_{k}$, we can rewrite the observable $y_{k}$ as

$$
\begin{aligned}
& y_{k} \simeq x_{k}+j \phi_{k} x_{k}+w_{k}=x_{k}+\left(j \phi_{k}\left|x_{k}\right|+w_{k}^{\prime}\right) e^{j \angle x_{k}} \\
& =x_{k}+\underbrace{\mathcal{R}\left\{w_{k}^{\prime}\right\} e^{j \angle x_{k}}}_{\text {radial noise }}+\underbrace{\left(\phi_{k}\left|x_{k}\right|+\mathcal{I}\left\{w_{k}^{\prime}\right\}\right) e^{j\left(\angle x_{k}+\frac{\pi}{2}\right)}}_{\text {angular noise }}
\end{aligned}
$$

where in (4) we have exploited the first order Taylor expansion $e^{j \phi_{k}} \simeq 1+j \phi_{k}$, which is good if $\phi_{k}$ is small, and $w_{k}^{\prime}$ is statistically equivalent to $w_{k}$. We can distinguish two different noise components, a radial noise term, with variance $\sigma_{w}^{2} / 2$, and an angular noise term, with variance

$$
\begin{aligned}
& \mathrm{E}\left[\left(\phi_{k}\left|x_{k}\right|+\mathcal{I}\left\{w_{k}^{\prime}\right\}\right)^{2}\right]=\frac{\sigma_{w}^{2}}{2}+\sigma_{\phi}^{2}\left|x_{k}\right|^{2} \\
& =\frac{\sigma_{w}^{2}}{2}\left(1+\left|x_{k}\right|^{2} \frac{2 \sigma_{\phi}^{2}}{\sigma_{w}^{2}}\right)=\frac{\sigma_{w}^{2}}{2}\left(1+f\left|x_{k}\right|^{2}\right),
\end{aligned}
$$

where we have defined $f$ as the ratio between the PN variance and the single-component AWGN variance. As we can see, the angular noise variance increases with the magnitude of the current symbol $x_{k}$. For this reason, a good constellation for a $\mathrm{PN}$ environment should increase the angular distance between points for increasing magnitude. We propose to increase the distance according to the standard deviation of the angular noise as

$$
D_{m} \simeq D \sqrt{1+f_{\mathrm{s}}\left|c_{m}\right|^{2}}
$$

where the parameter $f_{\mathrm{s}}$ can be chosen according to the channel conditions. One possible choice is to set $f_{\mathrm{s}}=f$. On the other hand, we have observed that better results can be achieved by a further tuning of this parameter by considering a proper performance metric, as we will discuss in details in the following sections. We define the parameters $t_{m}$ as

$$
t_{m}=\sqrt{4 \pi m \sqrt{1+f_{\mathrm{s}} t_{m}^{2}}},
$$

which, solving for $t_{m}^{2}$, becomes

$$
t_{m}^{2}=\frac{(4 \pi m)^{2} f_{\mathrm{s}}}{2}+\sqrt{\frac{(4 \pi m)^{4} f_{\mathrm{s}}^{2}}{4}+(4 \pi m)^{2}} .
$$

Finally, by replacing the expressions for $t_{m}$ in (3), we obtain the final expression for the constellation points. Notice that when $f_{\mathrm{s}}=0$ the expressions for the points reduce to those we obtained in Section III-A. The important aspect to underline here is that the proposed constellations ensure a very simple design, being dependent on a single parameter, as opposed to other multilevel constellations usually adopted in communication systems, such as APSKs, whose design requires the optimization of multiple parameters, namely the number of rings, the number of constellation points per ring, the amplitude and phase rotation of each ring.

\section{Performance Metrics}

In this section, we describe the figures of merit that we will employ to evaluate the performance of the proposed constellations, with the aim of finding both theoretical and more practical results. Regarding the detection approach, we consider the detector suitable for a PN scenario described in [3], based on an approximation of the received signal. As a benchmark to compare this detection scheme, we will use a detector that is designed for the AWGN channel, and hence does not take into account the presence of PN. Both solutions are based on the principle of mismatched detection [6], which assumes the use of a properly selected auxiliary channel to approximate the real channel under consideration. If an optimal detector is available for the auxiliary channel, the obtained results represent a lower bound on the performance of the real channel, achievable with the considered detector.

We will use different figures of merit. The first performance metric is the achievable information rate (IR), computed numerically through the Monte Carlo technique described in [7]. According to mismatched detection, this measure represents a lower bound to the channel capacity, which is achievable with joint detection and decoding, by using an optimum code. If we define by $\mathbf{x}=\left\{x_{k}\right\}_{k=0}^{K-1}$ a sequence of transmitted symbols, by $\mathbf{y}=\left\{y_{k}\right\}_{k=0}^{K-1}$ the corresponding received samples, and by $p(\mathbf{y} \mid \mathbf{x})$ the probability density function (PDF) of the selected auxiliary channel law, the lower bound on the achievable IR can be computed numerically as

$$
I(\mathbf{x} ; \mathbf{y})=\lim _{K \rightarrow \infty} \frac{1}{K} \mathrm{E}\left[\log _{2} \frac{p(\mathbf{y} \mid \mathbf{x})}{\sum_{\mathbf{x}^{\prime}} p\left(\mathbf{y} \mid \mathbf{x}^{\prime}\right) P\left(\mathbf{x}^{\prime}\right)}\right],
$$

where the summation at the denominator is performed over the $M$ symbols of the constellations, and $P\left(\mathbf{x}^{\prime}\right)$ is the probability distribution of the transmitted symbols, in this paper equal to $1 / M$ because the symbols are equally likely. Moreover, we will consider the channel to be memoryless, hence (8) can be simplified by replacing the vector PDF with its scalar 


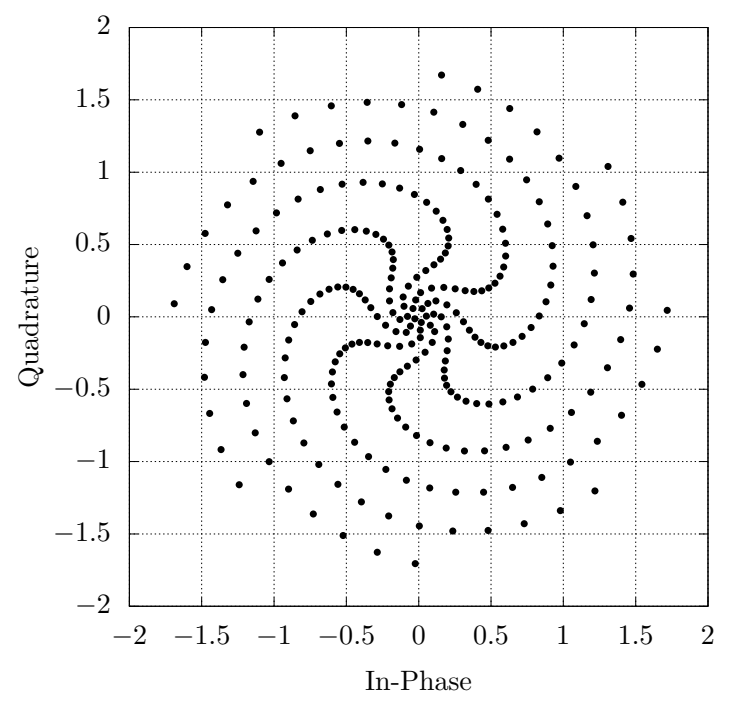

Figure 1. Spiral constellation with $M=256$ points and $f_{\mathrm{s}}=0.00413$.

version $p\left(y_{k} \mid x_{k}\right)$, which is representative of a symbol-bysymbol detector. The parameter $f_{\mathrm{s}}$ can be chosen as the one providing the largest IR. This search is carried out by evaluating (8) for several values of $f_{\mathrm{s}}$ of the spiral constellation (coarse search), followed by interpolation of the obtained values (fine search).

We will also consider an alternative theoretical measure, given by the pragmatic IR [8]. This represents an upper bound to the IR achieved by a practical modulation and coding format (ModCod) following a pragmatic approach, that is, without iterations between detector and decoder. The pragmatic IR depends on the adopted bits to symbols mapping, which controls how transmitted bits are mapped on the complex constellation symbols. Therefore, we will use this performance metric to optimize the parameter $f_{\mathrm{s}}$ given a particular bit to symbol mapping. The usual approach for PSKs and QAMs is Gray mapping, which ensures that adjacent constellation points differ by only one bit. APSKs usually adopt a quasiGray mapping, as they do not normally allow a fully Gray mapping. See for example those proposed in the extensions to the second generation of the digital video briadcasting (DVB$\mathrm{S} 2 \mathrm{X}$ ) standard [9]. When the constellation is not regular, a good mapping has to be found through optimization as well, possibly jointly with the constellation points [10]. In the case of the spiral constellations proposed in this work, we observed that points tend to form rays. When the number of rays is $\sqrt{M}$, we propose to map the columns of symbols of QAM modulations to the rays of the spiral. More generally, if the number of rays is a power of 2 , we can easily identify a transformation of the QAM symbols to map them into the rays of spiral. In Fig. 1, we show an example of a spiral with $M=256$ points and $f_{\mathrm{s}}=0.00413$. We can clearly see how the points are arranged in 8 rays, each composed of 32 points; in this case, it is easy to adapt two columns of the 256 QAM mapping to each of the rays, in order to achieve a mapping that is Gray along each ray and between adjacent rays.

Finally, we will compare these theoretical bounds with a more practical measure, given by the IR achieved by real coded schemes. To this purpose, we will evaluate by computer simulation the packet error rate of coded transmissions based on one of the low-density parity-check (LDPC) codes defined by the DVB-S2X standard [9], which have excellent performance on the AWGN channel. In this case, we use the parameter $f_{\mathrm{s}}$ optimized through the pragmatic IR.

\section{NumericAl RESUlts}

In this section, we present a set of numerical results and simulations regarding the application of spiral constellations in the considered PN environment. As a means of comparison, we consider the most commonly used multilevel modulation formats, namely QAM and APSK. For APSK, we adopt the ones proposed by the DVB-S2X standard [9], and the ones described in [11], which are designed to satisfy a Gray mapping. Using the same notation as in [11], a $\left(m_{1}, m_{2}\right)$ Gray APSK constellation is composed of $2^{m_{2}}$ rings, each containing $2^{m_{1}}$ equally spaced points; in this paper, we adopt the options, among those listed in [11], which ensure the best performance in presence of PN, namely the $(4,2) 64$ Gray APSK and the $(4,4) 256$ Gray APSK. We have also analyzed the spiral QAM constellations proposed in [5]. We consider, as an application example, constellations with $M=64$ and $M=256$ points. The same kind of analysis can be applied to any constellation size, and it is particularly interesting for large constellation sizes. All performance curves in this section are reported as a function of the signal-to-noise ratio (SNR), defined as the ratio between the average energy per symbol and the thermal noise variance, $E_{\mathrm{s}} / N_{0}$. When assessing the achievable IR for spiral constellations, an optimization is performed to select the best value of the parameter $f_{\mathrm{s}}$ for each $E_{\mathrm{s}} / N_{0}$ value. This choice is justified by the fact that, in common adaptive coding and modulation (ACM) schemes, the modulation and coding formats are changed according to the channel conditions.

First of all, we want to assess the performance of spiral constellations on the AWGN channel, that is, when the observable is modeled as in (1), with $\sigma_{\phi}^{2}=0$. Fig. 2 shows the achievable IR comparison in this case, for $M=64$ (a) and $M=256$ (b). We can notice that the spiral designed with $f_{\mathrm{s}}=0$ is essentially equivalent to QAM and to spiral QAM. On the other hand, an optimization of $f_{\mathrm{s}}$ can ensure theoretical gains up to almost $1 \mathrm{~dB}$ with respect to QAM constellations. The DVB-S2X APSKs show performance on par with the optimized spiral, except for small losses in the high SNR region, while Gray APSKs exhibit larger losses. This first analysis tells us that a properly designed spiral can achieve, on the AWGN channel, a theoretical performance that is slightly better than that of other commonly adopted constellations.

We then consider the channel affected by $\mathrm{PN}$. We use two values of PN variance, one relatively low, $\sigma_{\phi}^{2}=0.01$, representative of a well tracked $\mathrm{PN}$, and one 10 times higher, $\sigma_{\phi}^{2}=0.1$, corresponding to a poor tracking performance. The results, in terms of achievable IR, are reported in Fig. 3.

Let us first consider the $\sigma_{\phi}^{2}=0.01$ case. We first notice that the detector for AWGN, unsurprisingly, exhibits very 


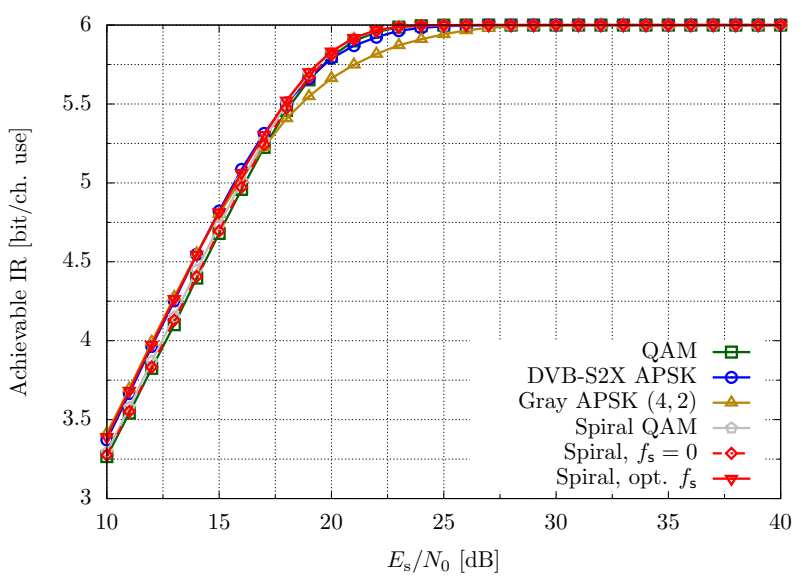

(a) $M=64, \sigma_{\phi}^{2}=0$

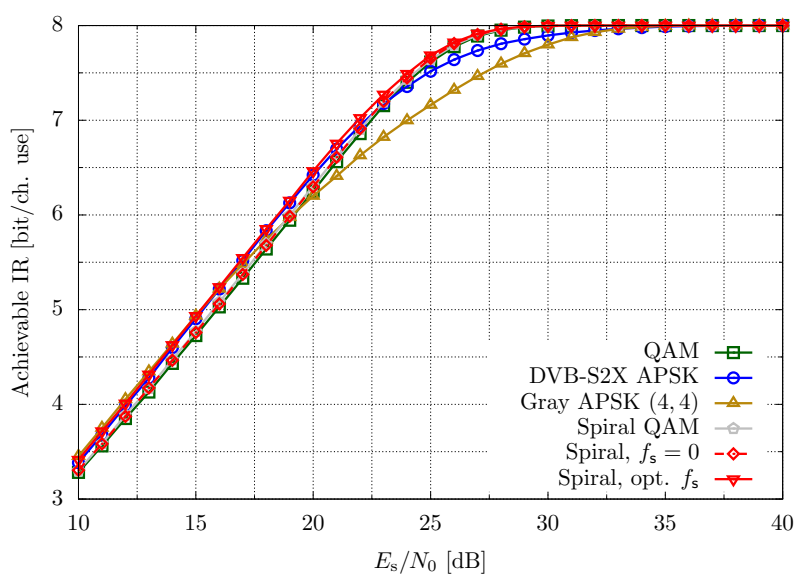

(b) $M=256, \sigma_{\phi}^{2}=0$

Figure 2. Achievable IR on the AWGN channel for constellations with 64 and 256 points.

poor performance if associated with QAMs, APSKs, and spiral QAMs ${ }^{1}$. Replacing the constellation with a suitable spiral, without changes at the receiver side, is sufficient to ensure a maximum achievable IR significantly higher than that achievable with the other constellations. The reference detector is clearly outperformed by the use of the alternative detection scheme, specifically designed for a PN environment. By comparing the different constellation types, we see that spirals ensure significant gains over QAMs and spiral QAMs, and that the gaps increase with the SNR. For example, at 7 bit/ch. use (with $M=256$ ), the gains are in the order of $3 \mathrm{~dB}$. APSKs outperform QAMs in most SNR regions, but are generally inferior to spirals, with the exception of the 256 Gray APSK, which is comparable with the spiral almost everywhere. The curves tend to coincide around $10 \mathrm{~dB}$, but it is worth to mention that an ACM scheme would probably adopt a lower constellation size in that SNR range, so the performance of higher order constellations is less relevant.

If we analyze the $\sigma_{\phi}^{2}=0.1$ case, instead, we see that

${ }^{1}$ The effect of the curves decreasing with the SNR is due to the adopted mismatched detection framework. In these SNR regions, PN is the main impairment, while the detector is designed for a completely different auxiliary channel, the AWGN channel. Hence, this detector is unable to work properly for these SNR values. spirals ensure much larger gains with respect to the other constellations. In this case, the best alternatives are a 64 spiral QAM and a 256 Gray APSK, which, however, suffer large losses at medium-high SNR. In this case we report only the curves obtained with the PN detector, the AWGN one being unable to work with this $\mathrm{PN}$ value.

Finally, if we compare the results in Fig. 3 with the corresponding curves for the AWGN channel reported in Fig. 2, we see that the difference is significant, and the gaps increase with the SNR. This is not surprising as, when the SNR increases, PN becomes the main impairment of the channel. However, we can notice that spiral constellations are the solution which offers the best performance in terms of achievable IR in all channel conditions. These results show that the spiral QAM constellations proposed in [5] exhibit significant losses with respect to our proposed spirals in all PN conditions. Moreover, their design process does not allow for a simple mathematical formulation and, instead, relies on a more computationally intensive procedure. For these reasons, we will not consider them further.

Fig. 4 reports the pragmatic IR curves for the same cases as in Fig. 3, adopting only the detector for PN. For $\sigma_{\phi}^{2}=$ 0.01 , we can notice that in the medium-low SNR range Gray APSKs provide the best performance, outperforming spirals and QAMs of at most $1 \mathrm{~dB}$ for $M=64$ and $2 \mathrm{~dB}$ for $M=$ 256. Also in this case, spirals outperform all alternatives at high SNR, for $M=64$, and are comparable to the 256 Gray APSK. The loss of the spiral constellations is probably due to the suboptimality of the adopted bits to symbols mapping, and could be recovered with a more complex mapping obtained through optimization. For $\sigma_{\phi}^{2}=0.1$, spirals show extremely large gains starting from lower SNR values, so they certainly represent the desirable solution.

In order to confirm these theoretical results, we have selected one of the LDPC codes from the DVB-S2X standard [9], with codeword size 64800 bits and rate $r=5 / 6$. The results are reported in Table I, where the achieved IR (3rd column) is computed as $r \log _{2} M$, and it represents the number of bits that can be effectively transferred in a single use of the channel. The SNR columns of the table contain the values of $E_{\mathrm{s}} / N_{0}$ needed to achieve a bit error rate (BER) of $10^{-6}$. When this value is $\infty$, it means that the BER never reaches sufficiently low values. The table also reports the gaps of each ModCod from the corresponding achievable and pragmatic IR, and the peak to average power ratio of each constellation (PAPR), which is another crucial measurement for wireless communications, where high power amplifiers are used. The values of $f_{\mathrm{s}}$ for the spiral constellations are those selected by means of the technique described in Section IV. By analyzing the table, we see that the results are perfectly in line with what foreseen by the pragmatic IR. In particular, if we look at the $\sigma_{\phi}^{2}=0.01$ case, we see that the 64 Gray APSK exhibits a $0.4 \mathrm{~dB}$ gain over the spiral, with a comparable PAPR, and that both QAM and DVB-S2X APSK have a lower PAPR and slightly worse performance, comparable to that of the spiral. For $M=256$, instead, the Gray APSK shows a $0.9 \mathrm{~dB}$ 


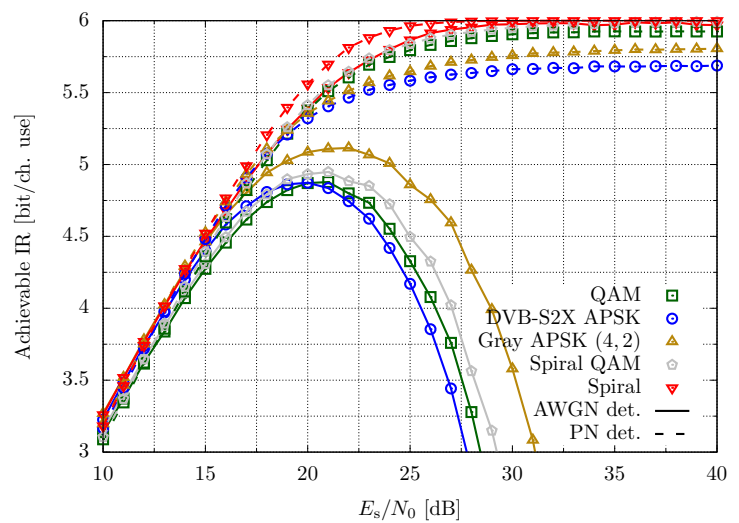

(a) $M=64, \sigma_{\phi}^{2}=0.01$

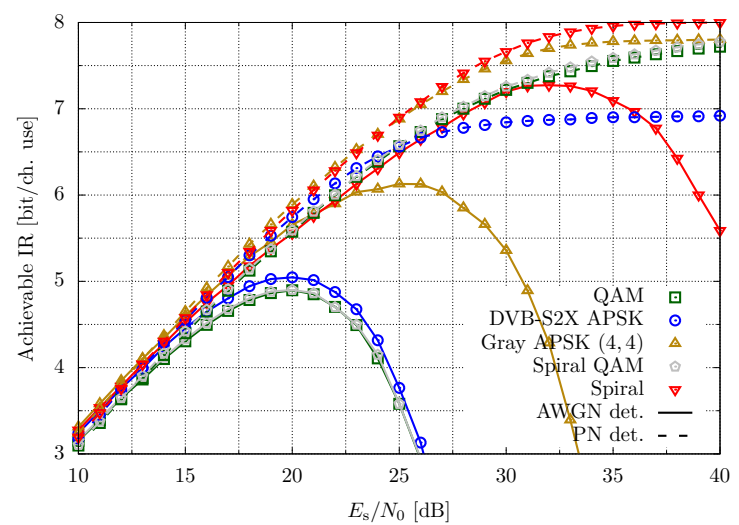

(c) $M=256, \sigma_{\phi}^{2}=0.01$

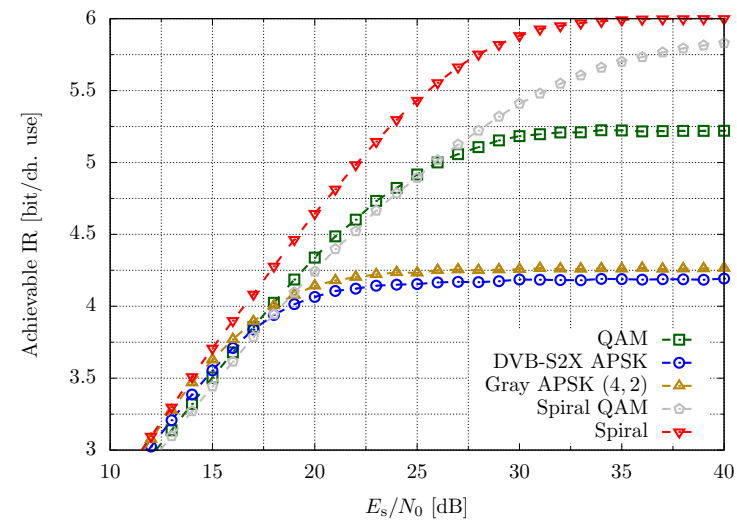

(b) $M=64, \sigma_{\phi}^{2}=0.1$

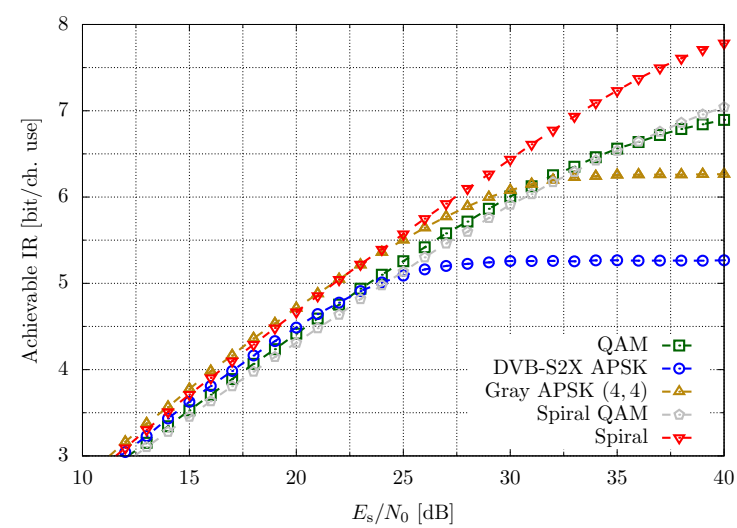

(d) $M=256, \sigma_{\phi}^{2}=0.1$

Figure 3. Achievable IR on the channels affected by PN, for constellations with 64 and 256 points.

performance gain with respect to the spiral, with a $0.65 \mathrm{~dB}$ higher PAPR. Both the QAM and the DVB-S2X APSK show poor convergence in this case. In the $\sigma_{\phi}^{2}=0.1$ case, on the other hand, spiral constellations are the only solution which is able to converge, for both $M=64$ and $M=256$, and the gaps from the corresponding pragmatic IR are not excessive, despite the fact that the adopted code has been specifically optimized for the AWGN channel. Notice that the constellation reported in Fig. 1 is used in this case.

\section{Conclusions}

We have proposed new spiral constellations, specifically designed for phase noise channels. The points of the new constellations are described by an elegant analytical form, that allows an extremely simple design. In terms of performance, they outperform other common constellations for a large range of SNR, especially in harsh phase noise conditions. Moreover, we have shown that they allow to achieve high information rate, close to 8 bits per channel use, and they are suitable for application in practical coded systems adopting state-of-the-art channel codes, without the need for a redesign of the code.

\section{APPENDIX A}

\section{ProOF OF THEOREM 1}

Proof: We approximate the distance between two points on the spiral by the distance along a circle at the average radius, which is given by

$$
D_{m} \simeq t_{m}\left(t_{m+1}-t_{m}\right)
$$

If we replace the expression for the constellation points, $t_{m}=$ $\sqrt{2 D m}$, in the distance equation (9), we obtain

$$
\begin{aligned}
D_{m} & =\sqrt{2 D m}(\sqrt{2 D m+2 D}-\sqrt{2 D m})=2 D m\left(\sqrt{1+\frac{1}{m}}-1\right) \\
& \simeq 2 D m\left(1+\frac{1}{2 m}-\frac{1}{8 m^{2}}-1\right)=D\left(1-\frac{1}{4 m}\right) \simeq D .
\end{aligned}
$$

The approximation is good for large values of $m$. Now, we also want the laps of the spiral (perpendicular to the spiral) to be at the same distance $D$ as the distances along the spiral. So, if we move a full lap, the magnitude should be increased by $D$, according to the following equation,

$$
\left|(t+2 \pi) e^{j(t+2 \pi)}\right|=\left|t e^{j t}\right|+D,
$$

from which $D=2 \pi$ follows. For small values of $m$, the distances between points deviate slightly from the desired constant $2 \pi$.

\section{REFERENCES}

[1] G. Foschini, R. Gitlin, and S. Weinstein, "On the selection of a twodimensional signal constellation in the presence of phase jitter and Gaussian noise,” Bell System Tech. J., vol. 52, pp. 927-965, July 1973. 


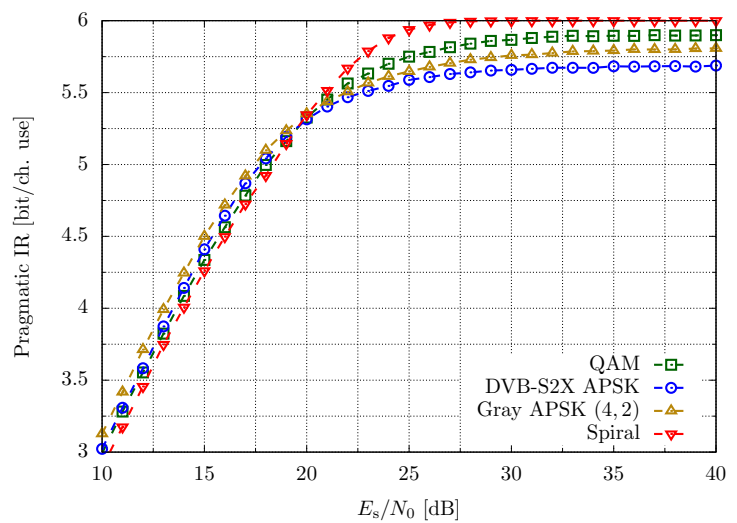

(a) $M=64, \sigma_{\phi}^{2}=0.01$

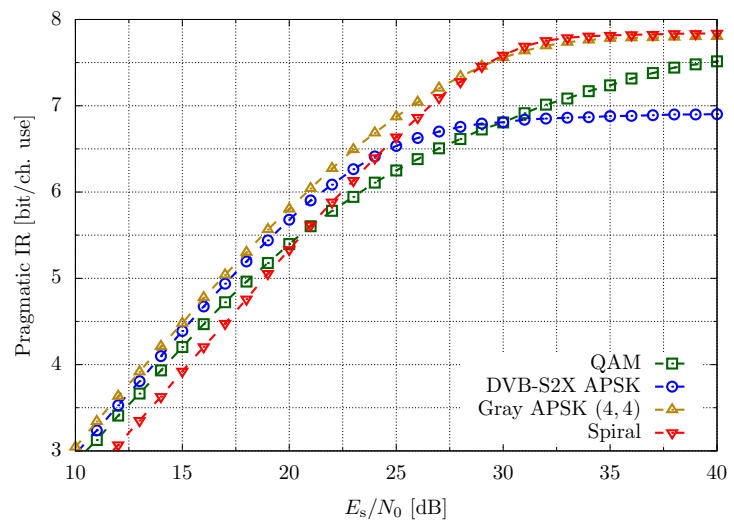

(c) $M=256, \sigma_{\phi}^{2}=0.01$

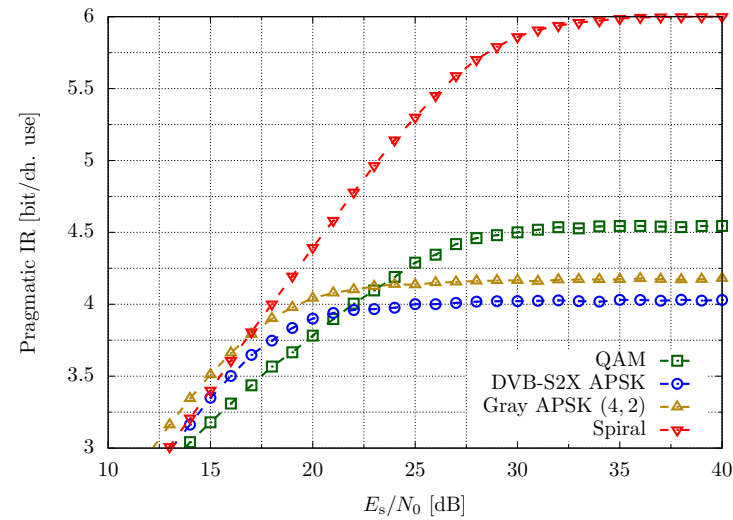

(b) $M=64, \sigma_{\phi}^{2}=0.1$

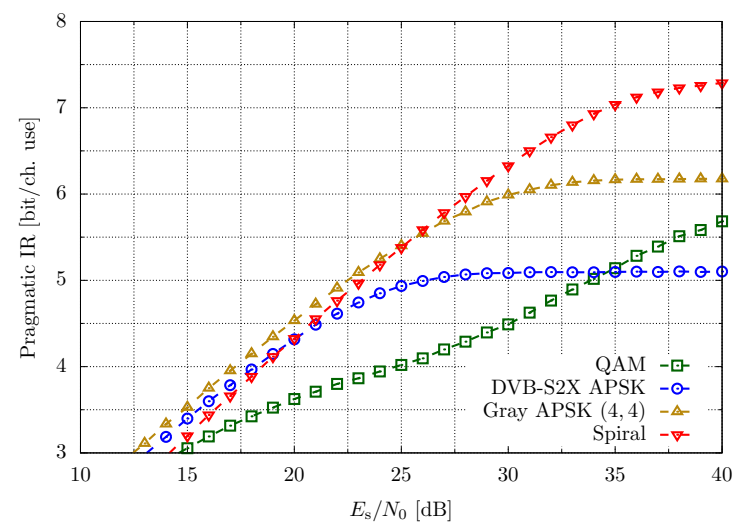

(d) $M=256, \sigma_{\phi}^{2}=0.1$

Figure 4. Pragmatic IR on the channels affected by PN, for constellations with 64 and 256 points.

Table I

MODCODS PERFORMANCE.

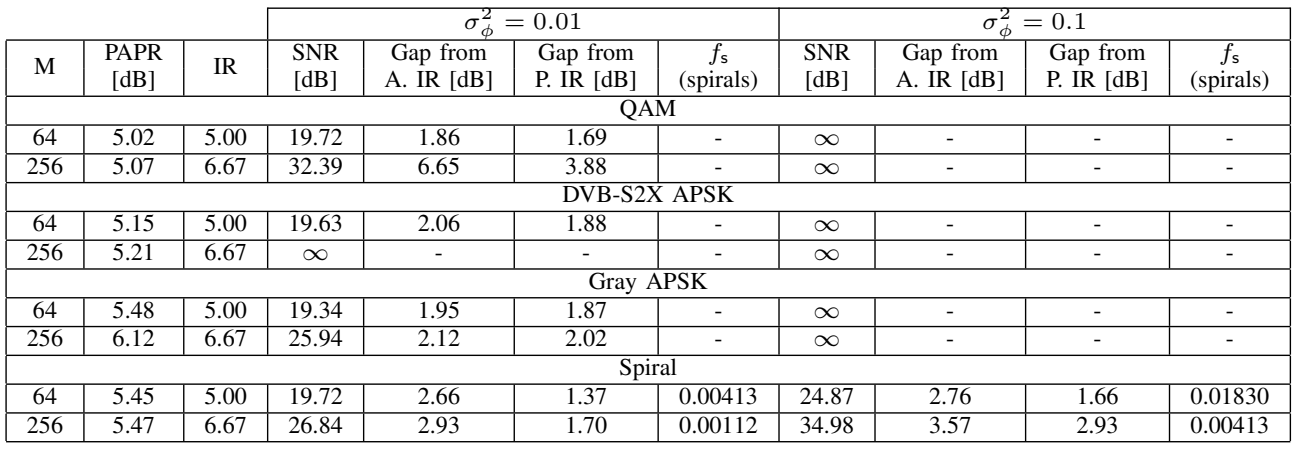

[2] F. Kayhan and G. Montorsi, "Constellation design for memoryless phase noise channels," IEEE Trans. Wireless Commun., vol. 13, pp. 2874-2883, May 2014.

[3] R. Krishnan, A. Graell i Amat, T. Eriksson, and G. Colavolpe, "Constellation optimization in the presence of strong phase noise," IEEE Trans. Commun., vol. 61, pp. 5056-5066, Dec. 2013.

[4] M. A. Tariq, H. Mehrpouyan, and T. Svensson, "Performance of circular QAM constellations with time varying phase noise," in Proc. IEEE International Symposium on Personal, Indoor, and Mobile Radio Commun., (Sydney, Australia), pp. 2365-2370, Sept. 2012.

[5] B. J. Kwak, N. O. Song, B. Park, and D. S. Kwon, "Spiral QAM: A novel modulation scheme robust in the presence of phase noise," in Proc. Vehicular Tech. Conf., (Calgary, BC, Canada), Sept. 2008.

[6] N. Merhav, G. Kaplan, A. Lapidoth, and S. Shamai, "On information rates for mismatched decoders," IEEE Trans. Inform. Theory, vol. 40, pp. 1953-1967, Nov. 1994.

"Simulation-based computation of information rates for channels with

[7] D. M. Arnold, H.-A. Loeliger, P. O. Vontobel, A. Kavčić, and W. Zeng, memory," IEEE Trans. Inform. Theory, vol. 52, pp. 3498-3508, Aug. 2006.

[8] J. B. Soriaga, H. Pfister, and P. Siegel, "Determining and approaching achievable rates of binary intersymbol interference channels using multistage decoding," IEEE Trans. Inform. Theory, vol. 53, pp. 1416-1429, Apr. 2007.

[9] ETSI EN 302 307-2 Digital Video Broadcasting (DVB), Second generation framing structure, channel coding and modulation systems for Broadcasting, Interactive Services, News Gathering and other broadband satellite applications, Part II: S2-Extensions (DVB-S2X). Available on ETSI web site (http://www.etsi.org).

[10] F. Kayhan and G. Montorsi, "Joint signal-labeling optimization under peak power constraint," Intern. J. of Satellite Communications and Networking, vol. 30, pp. 251-263, Nov./Dec. 2012.

[11] Z. Liu, Q. Xie, K. Peng, and Z. Yang, "APSK constellation with Gray mapping," IEEE Commun. Letters, vol. 15, pp. 1271-1273, Dec. 2011. 